\title{
Estudio preliminar de la flora liquénica de Isla Mocha, sur de Chile
}

\section{Preliminary study of the lichen flora of Isla Mocha, southern Chile}

\author{
Wanda Quilhot, Mauricio Cuellar, Rodrigo Díaz, Francisco Riquelme \& Cecilia Rubio \\ Herbario de Líquenes, Departamento de Química y Recursos Naturales, Facultad de Farmacia, Universidad de Valparaíso, \\ Casilla 5001, Valparaíso, Chile. \\ wanda.quilhot@uv.cl
}

\begin{abstract}
RESUMEN
La micobiota liquenizada de Isla Mocha, sur de Chile, está representada por 76 especies en 30 géneros; el $38 \%$ de las especies son endémicas del sur de Sudamérica. Xanthoria parietina, registrada en todas las zonas de muestreo, y Pseudocyphellaria malmeana recolectada sólo en el bosque de Aextoxicon punctatum, son las especies más representativas. Ramalina fastigiata constituye un nuevo registro para Chile.
\end{abstract}

Palabras clave: Líquenes, distribución, endemismo, Aextoxicon punctatum.

\begin{abstract}
The lichenized mycobiota of Isla Mocha, southern Chile, includes 76 taxa in 30 genera; $38 \%$ of the species are endemic to southern South America. Xanthoria parietina, found in all the collecting zones, and Pseudocyphellaria malmeana growing only on bark of Aextoxicon punctatum, are the most representative species. Ramalina fastigiata is a new record to Chile.
\end{abstract}

KEYWORDS: Lichen, distribution, endemism, Aextoxicon punctatum.

\section{INTRODUCCIÓN}

Chile tiene una rica y variada flora liquénica en términos de diversidad y biomasa; sin embargo el conocimiento de los líquenes es aún incompleto, probablemente por el escaso número de especialistas y porque aún existen en el país zonas no exploradas.

Desde la publicación de la Lista Patrón de los Líquenes y Hongos Liquenolícolas de Chile (Galloway \& Quilhot 1998) que incluye un total de 1.383 taxones, la riqueza específica ha aumentado a 1.454 taxones debido, principalmente, a la intensidad de la exploración. Se han descrito nuevas especies (e.g. Jørgensen 1998, Jørgensen \& Wedin 1999, Bjerke 2001, Bjerke et al. 2003 ab, Bjerke 2005, Elvebakk \& Galloway 2003, Elvebakk \& Bjerke 2005, Galloway et al. 2005, Lücking et al. 2003) así como nuevos registros para el país (eg. Lücking et al. 2003, Galloway et al. 2006, Pereira \& Torres 2005, Pereira 2007, Hertel 2007, Kalb et al. 2008).

El propósito de este estudio fue investigar la diversidad liquénica en Isla Mocha, ubicada a los $38^{\circ}$ en el sur de Chile.
Es un área natural con comunidades vegetales que presentan una estrecha afinidad florística con la biota continental y que, desde un punto de vista biogeográfico, se relacionan con los bosques de neblina del Norte Chico y con el bosque templado valdiviano (Le Quesne et al. 1999, Mendoza \& Jerez 2001). Incluye la Reserva Forestal Isla Mocha dominada por el bosque relicto de Aextoxicon punctatum Ruiz \& Pav., además de especies como Peumus boldus Molina, Drimys winteri J.R. Forst \& G. Forst, Fuchsia magellanica Lam., Aristotelia chilensis Stuntz, Luma apiculata (DC.) Burret, Laureliopsis philippiana (Looser) Schode, Myrceugenia planipes (Hook \& Arn.) O. Berg (Le Quesne et al. 1999). Por su aislamiento geográfico, la Isla Mocha puede ser considerada como una micro-ecorregión porque reúne un amplio número de especies en condiciones ambientales similares y cuyas interacciones ecológicas son críticas para su permanencia en el largo plazo (Dinerstein et al. 1995).

La floraliquénica de Isla Mochano había sidoinvestigada; sólo se conocía la presencia de Pseudocyphellaria 
malmeana, P. flavicans y P. divulsa (Galloway 1992) en este bioma.

\section{MATERIALES Y MÉTODOS}

La Isla Mocha ( $38^{\circ} 23^{\prime} \mathrm{S} ; 73^{\circ} 54^{\prime} \mathrm{W}$ ) está ubicada en el golfo de Arauco, en el límite de la Región del Bío-Bío y el límite de la Región de la Araucanía, a $35 \mathrm{~km}$ del pueblo de Tirúa. $\mathrm{Su}$ extensión es de $13 \mathrm{~km}$ de largo y $6 \mathrm{~km}$ de ancho y su superficie es de $48 \mathrm{~km}^{2}$; la altura fluctúa entre el nivel del mar y $349 \mathrm{~m}$.

Las recolecciones se hicieron en cuatro zonas seleccionadas, luego de un reconocimiento del terreno (Fig. 1), en octubre de 2008 y octubre de 2009 , y corresponden a las siguientes:

Zona 1: (38 $\left.24^{\prime} \mathrm{S} ; 7^{\circ} 54^{\prime} \mathrm{W}\right)$ ubicada entre la franja intermedia y el borde del bosque, es un área en la que predominan Peumus boldus, Aristotelia chilensis, Luma apiculata y Laureliopsis philippiana.

Zona 2: (38 $\left.21^{\prime} \mathrm{S} ; 7^{\circ} 55^{\prime} \mathrm{W}\right)$ ubicada en el bosque relicto de Aextoxicon punctatum; las especies acompañantes incluyen, entre otras, a Aristotelia chilensis, Luma apiculata, Laureliopsis philippiana, Drimys winteri. Los muestreos se hicieron al interior del bosque, entre 50 y 324 ms.n.m.

Zona 3: (38 $22^{\prime} \mathrm{S}$; $\left.73^{\circ} 55^{\prime} \mathrm{W}\right)$ ubicada en la franja intermedia, con predominancia de Peumus boldus y algunas especies arbustivas acompañantes, frente al mar y atravesada por el camino vecinal.

Zona 4: (38 $22^{\circ}$ 'S; $\left.73^{\circ} 56^{\prime} \mathrm{W}\right)$ ubicada en la franja costera, en la Península Piedra Antigua, a 6 ms.n.m y expuesta a la influencia de las mareas.

Las observaciones anatómicas y morfológicas del material liquénico se realizaron utilizando una lupa estereoscópica Leica DMLS y un microscopio Nikon modelo 104, en secciones de talo muy delgadas cortadas a mano y montadas en agua; en algunas preparaciones se utilizó azul de metileno como medio de tinción.

El material recolectado se encuentra depositado en el Herbario de Líquenes (UV) de la Facultad de Farmacia, Universidad de Valparaíso. Los autores de los taxones están citados en la Tabla I que incluye sólo el material determinado a nivel de especie, y la nomenclatura sigue a Galloway \& Quilhot (1998).

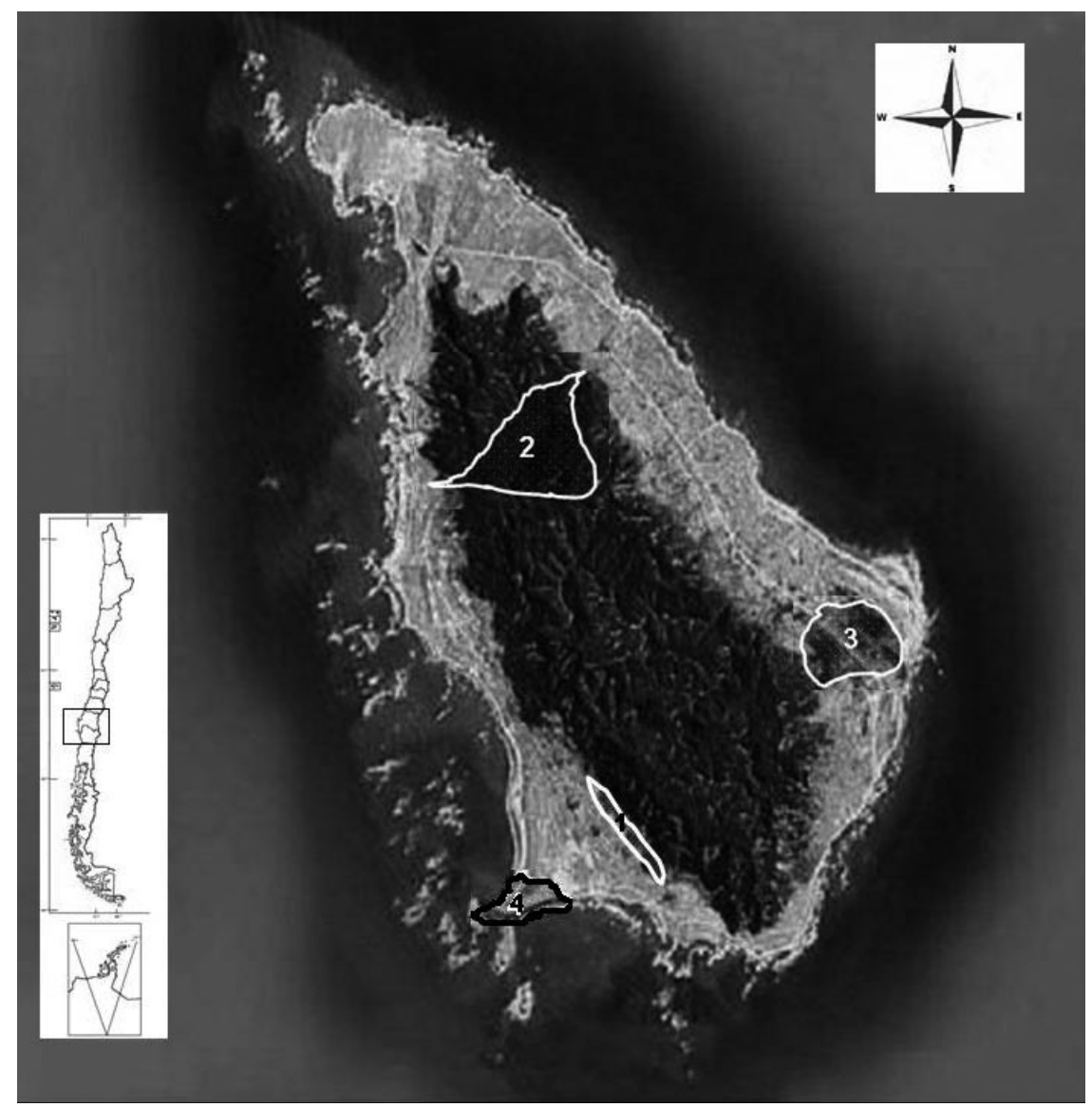

FIGURA 1. Ubicación de las zonas de muestreo en Isla Mocha. (imagen satelital extraída de Google Earth ${ }^{\mathrm{MR}}$ ).

FIGURE 1. Location of the collection zones in Isla Mocha. (satelital image from Google Earth ${ }^{\mathrm{TM}}$ ). 
TABLa I. Líquenes de Isla Mocha.

TABLE I. Lichens of Isla Mocha.

\begin{tabular}{|c|c|c|c|c|c|}
\hline \multirow{2}{*}{ ESPECIE } & \multicolumn{3}{|c|}{ ZONAS } & \multirow{2}{*}{ PATRONES DE DISTRIBUCIÓN } & \multirow{2}{*}{ REFERENCIAS } \\
\hline & 1 & 2 & 3 & & \\
\hline Candelaria concolor (Dickson) B.Stein. & & & - & Cosmopolita & Galloway 1985, Wetsberg \& Nash III 2002 \\
\hline Chrysothrix pavonii (Fr.) J.R.Laundon & & - & - & Cosmopolita & Laundon 1981 \\
\hline Coccotrema cucurbitula (Mont.) Müll. Arg. & & & - & Austral & Messuti 1996 \\
\hline Collema laeve Hook.f. \& Taylor & & - & & Australásico & Filson 1992 \\
\hline $\begin{array}{l}\text { Coenogonium luteum (Dicks.) Kalb \& } \\
\text { Lücking }\end{array}$ & & - & & Tropical & $\begin{array}{l}\text { Lücking \& Kalb (2000), Rivas Plata et al. } \\
2006\end{array}$ \\
\hline Degeliella versicolor (Müll.Arg.) P.M.Jørg. & & & - & Austral & Jørgensen 2004 \\
\hline $\begin{array}{l}\text { Dictyonema glabratum (Spreng.) } \\
\text { D.Hawksw. }\end{array}$ & & - & & Pantropical & Parmasto 1978, Hawksworth 1988 \\
\hline $\begin{array}{l}\text { Flavoparmelia rutidota (Hook.f. \& Taylor) } \\
\text { Hale }\end{array}$ & & & - & $\begin{array}{l}\text { Norte y Sudamérica, } \\
\text { Australásico }\end{array}$ & Nash III \& Elix 2002a \\
\hline Flavoparmelia soredians (Nyl.) Hale & & - & - & Cosmopolita & Hale 1986 \\
\hline Hypogymnia subphysodes (Kremp.) Filson & & - & & Austral & Elix 1992 \\
\hline Hypotrachyna brevirhiza (Kurok.) Hale & & & - & Pantropical & Hale 1975 \\
\hline Hypotrachyna laevigata $(\mathrm{Sm}$.$) Hale$ & & - & & Cosmopolita & Hale 1975 \\
\hline $\begin{array}{l}\text { Leptogium australe (Hook.f. \& Taylor) } \\
\text { Müll.Arg. }\end{array}$ & & - & - & $\begin{array}{l}\text { Aparentemente endémico } \\
\text { del sur de Sudamérica }\end{array}$ & Galloway \& Jørgensen 1995 \\
\hline Leptogium azureum (Sw.) Mont. & & - & - & $\begin{array}{l}\text { Endémico del sur de } \\
\text { Sudamérica }\end{array}$ & Galloway \& Jørgensen 1995 \\
\hline Leptogium brebissonii Mont. & & - & - & $\begin{array}{l}\text { África del Este, Europa, } \\
\text { Macronesia }\end{array}$ & Galloway \& Jørgensen 1995 \\
\hline $\begin{array}{l}\text { Leptogium britannicum P.M.Jørg. \& } \\
\text { P.James }\end{array}$ & - & & - & Bipolar & Galloway \& Jørgensen 1995 \\
\hline $\begin{array}{l}\text { Leptogium cochleatum (Dicks.) P.M.Jørg. } \\
\& \text { P.James }\end{array}$ & & & - & Subtropical & Galloway \& Jørgensen 1995 \\
\hline $\begin{array}{l}\text { Leptogium coralloideum (Meyen \& Flot.) } \\
\text { Vain. }\end{array}$ & & - & - & Tropical-temperado & Galloway \& Jørgensen 1995 \\
\hline Leptogium decipiens P.M.Jørg. & & & - & $\begin{array}{l}\text { Endémico del sur de } \\
\text { Sudamérica }\end{array}$ & Galloway \& Jørgensen 1995 \\
\hline Leptogium menziesii (Ach.) Mont. & & - & & Sudamericano & Galloway \& Jørgensen 1995 \\
\hline $\begin{array}{l}\text { Menegazzia fumarprotocetrarica Calvelo } \\
\& \text { Adler }\end{array}$ & & & - & $\begin{array}{l}\text { Endémico del sur de } \\
\text { Sudamérica }\end{array}$ & Adler \& Calvelo 1996; Bjerke et al. 2003a \\
\hline Menegazzia globulifera R.Sant. & & - & - & Tropical-temperado & Bjerke et al. 2003b \\
\hline Menegazzia neozelandica (Zahlbr.) P.James & & & - & $\begin{array}{l}\text { Argentina, Tasmania, N. } \\
\text { Zelanda }\end{array}$ & Bjerke et al. 2003b \\
\hline Menegazzia norsorediata Adler \& Calvelo & & & - & $\begin{array}{l}\text { Endémico del sur de } \\
\text { Sudamérica }\end{array}$ & Adler \& Calvelo 1996, Bjerke et al. 2003b \\
\hline Menegazzia violascens (Räsänen) Bjerke & & & - & $\begin{array}{l}\text { Endémico del sur de } \\
\text { Sudamérica }\end{array}$ & Bjerke 2005 \\
\hline Menegazzia wandae Bjerke & & & - & $\begin{array}{l}\text { Endémico del sur de } \\
\text { Sudamérica }\end{array}$ & Bjerke 2001 \\
\hline Nephroma antarcticum (Jacq.) Nyl. & & - & - & $\begin{array}{l}\text { Endémico del sur de } \\
\text { Sudamérica }\end{array}$ & White \& James 1988 \\
\hline Nephroma plumbeum (Mont.) Mont. & - & - & & Austral & White \& James 1988 \\
\hline Normandina pulchella (Borrer) Nyl. & & & - & Cosmopolita & Galloway 1985 \\
\hline $\begin{array}{l}\text { Pannaria durietzii (P.James \& Henssen) } \\
\text { Elvebakk \& D.J.Galloway }\end{array}$ & & & - & Australásico & Elvebakk \& Galloway 2003 \\
\hline $\begin{array}{l}\text { Pannaria isabellina (Vain.) Elvebakk \& } \\
\text { Bjerke }\end{array}$ & & & - & $\begin{array}{l}\text { Endémico del sur de } \\
\text { Sudamérica }\end{array}$ & Elvebakk \& Bjerke 2005 \\
\hline Peltigera didactyla (With.) J.R.Laundon & & & - & Cosmopolita & Martínez et al. 2003 \\
\hline Phlyctis chilensis D.J.Galloway \& Guzmán & & - & - & $\begin{array}{l}\text { Endémico del sur de } \\
\text { Sudamérica }\end{array}$ & Galloway \& Guzmán 1988 \\
\hline Physcia aipolia (Ehrh. ex Humb.) Fürnr. & & & - & Cosmopolita & Galloway 1985 \\
\hline Pseudocyphellaria argyracea (Delise) Vain. & & - & & Paleotropical & Galloway 1992 \\
\hline Pseudocyphellaria bartlettii D.J.Galloway & & - & & Paleotropical & Galloway 1992 \\
\hline $\begin{array}{l}\text { Pseudocyphellaria berberina (G.Forster) } \\
\text { D.J.Galloway \& P. James }\end{array}$ & - & - & - & $\begin{array}{l}\text { Endémico del sur de } \\
\text { Sudamérica }\end{array}$ & Galloway 1992 \\
\hline
\end{tabular}




\begin{tabular}{|c|c|c|c|c|}
\hline Pseudocyphellaria compar (Nyl.) H.Magn. & - & - & $\begin{array}{l}\text { Endémico del sur de } \\
\text { Sudamérica }\end{array}$ & Galloway 1992 \\
\hline $\begin{array}{l}\text { Pseudocyphellaria coriifolia (Müll.Arg.) } \\
\text { Malme }\end{array}$ & - & & $\begin{array}{l}\text { Endémico del sur de } \\
\text { Sudamérica }\end{array}$ & Galloway 1992 \\
\hline Pseudocyphellaria crocata (L.) Vain. & - & - & Cosmopolita & Galloway 1992 \\
\hline $\begin{array}{l}\text { Pseudocyphellaria dissimilis (Nyl.) } \\
\text { D.J.Galloway \& P.James }\end{array}$ & - & & $\begin{array}{l}\text { Endémico del sur de } \\
\text { Sudamérica }\end{array}$ & Galloway 1992 \\
\hline $\begin{array}{l}\text { Pseudocyphellaria exanthematica I.M. } \\
\text { Lamb }\end{array}$ & - & - & $\begin{array}{l}\text { Endémico del sur de } \\
\text { Sudamérica }\end{array}$ & Galloway 1992 \\
\hline $\begin{array}{l}\text { Pseudocyphellaria flavicans (Hook.f. \& } \\
\text { Taylor) Vain. }\end{array}$ & - & & $\begin{array}{l}\text { Endémico del sur de } \\
\text { Sudamérica }\end{array}$ & Galloway 1992 \\
\hline $\begin{array}{l}\text { Pseudocyphellaria glabra (Hook.f. \& } \\
\text { Taylor) C.W.Dodge }\end{array}$ & - & - & Austral & Galloway 1992 \\
\hline Pseudocyphellaria intricata (Delise) Vain. & - & a & Cosmopolita & Galloway 1992 \\
\hline Pseudocyphellaria malmeana D.J.Galloway & - & & $\begin{array}{l}\text { Endémico del sur de } \\
\text { Sudamérica }\end{array}$ & Galloway 1992 \\
\hline $\begin{array}{l}\text { Pseudocyphellaria meyenii (Trevis.) } \\
\text { D.J.Galloway }\end{array}$ & - & - & $\begin{array}{l}\text { Endémico del sur de } \\
\text { Sudamérica }\end{array}$ & Galloway 1992 \\
\hline Pseudocyphellaria nitida (Taylor) Malme & - & & $\begin{array}{l}\text { Endémico del sur de } \\
\text { Sudamérica }\end{array}$ & Galloway 1992 \\
\hline $\begin{array}{l}\text { Pseudocyphellaria norvegica (Gyeln.) } \\
\text { P.James }\end{array}$ & - & & Cosmopolita & Galloway 1992 \\
\hline Pseudocyphellaria pluvialis R.Sant. & - & & $\begin{array}{l}\text { Endémico del sur de } \\
\text { Sudamérica }\end{array}$ & Galloway 1992 \\
\hline $\begin{array}{l}\text { Pseudocyphellaria santessonii } \\
\text { D.J.Galloway }\end{array}$ & - & - & $\begin{array}{l}\text { Endémico del sur de } \\
\text { Sudamérica }\end{array}$ & Galloway 1992 \\
\hline Pseudocyphellaria scabrosa R.Sant. & - & & $\begin{array}{l}\text { Endémico del sur de } \\
\text { Sudamérica }\end{array}$ & Galloway 1992 \\
\hline $\begin{array}{l}\text { Pseudocyphellaria valdiviana (Nyl.) } \\
\text { Follmann }\end{array}$ & - & & $\begin{array}{l}\text { Endémico del sur de } \\
\text { Sudamérica }\end{array}$ & Galloway 1992 \\
\hline Psoroma caliginosum Stirt. & घ & & Austral & Lücking et al. 2003 \\
\hline Psoroma hypnorum (Vahl) S.F.Gray & & - & Cosmopolita & $\begin{array}{l}\text { Arvidsson \& Galloway 1992, Øvstedal \& } \\
\text { Lewis Smith } 2001\end{array}$ \\
\hline Punctelia borreri (Sm.) Krog & घ & - & Cosmopolita & Galloway 1985 \\
\hline Punctelia subrudecta (Nyl.) Krog & घ & a & Cosmopolita & Galloway 1985 \\
\hline $\begin{array}{l}\text { Ramalina celastri (Spreng.) Krog \& } \\
\text { Swinscow }\end{array}$ & - & - & Paleotropical & Kashiwadani 1990 \\
\hline Ramalina chilena (Nyl.) Kashiw. & - & - & $\begin{array}{l}\text { Aparentemente endémico } \\
\text { del sur de Sudamérica }\end{array}$ & Kashiwadani 1990 \\
\hline Ramalina chilensis Bertero ex.Nyl. & - & - & $\begin{array}{l}\text { Endémico del sur de } \\
\text { Sudamérica }\end{array}$ & Follmann 1967 \\
\hline Ramalina farinacea (L.) Ach. & & - & Circumpolar & Kashiwadani 1990, Goward \& Ahti 1992 \\
\hline Ramalina fastigiata (Pers.) Ach. & & - & Subcosmopolita & Crespo \& Bueno 1984 \\
\hline Rimelia reticulata (Taylor) Hale \& Fletcher & घ & - & Subtropical-pantemperado & Nash III \& Elix 2002b \\
\hline Roccellina limitata (Nyl.) Tehler & & - & Sudamericano & Tehler 1983 \\
\hline Sticta ainoae D.J.Galloway \& J.Pickering & - & - & $\begin{array}{l}\text { Endémico del sur de } \\
\text { Sudamérica }\end{array}$ & Galloway 1994 \\
\hline Sticta caulescens De Not. & - & & $\begin{array}{l}\text { Endémico del sur de } \\
\text { Sudamérica }\end{array}$ & Galloway 1994 \\
\hline Sticta lineariloba (Mont.) Nyl. & & a & $\begin{array}{l}\text { Endémico del sur de } \\
\text { Sudamérica }\end{array}$ & Galloway 1994 \\
\hline Sticta longipes (Müll. Arg.) Malme & - & & $\begin{array}{l}\text { Endémico del sur de } \\
\text { Sudamérica }\end{array}$ & Galloway 1994 \\
\hline $\begin{array}{l}\text { Sticta sublimbata (Steiner) Swinscow \& } \\
\text { Krog }\end{array}$ & - & - & Africa del Este, Caribe & Galloway 1994 \\
\hline Teloschistes chrysophthalmus (L.) Th.Fr. & घ & - & Cosmopolita & Galloway 1985 \\
\hline Teloschistes flavicans (Sw.) Norman & - & - & Cosmopolita & Galloway 1985 \\
\hline Teloschistes velifer F.Wils. & - & - & Australásico-paleotropical & Galloway 1985 \\
\hline Thelotrema lepadinum (Ach.) Ach. & - & & Cosmopolita & Galloway 1985 \\
\hline Usnea pusilla (Räsänen) Räsänen & - & - & Austral & Galloway 1985 \\
\hline Usnea rubicunda Stirt. & - & - & Cosmopolita & Galloway 1985 \\
\hline Xanthoria parietina $($ L.) Th.Fr. & घ & a & Cosmopolita & Galloway 1985 \\
\hline
\end{tabular}




\section{RESULTADOS Y DISCUSIÓN}

Se registraron 76 taxones pertenecientes a 30 géneros (Tabla I) que colonizan diferentes sustratos tales como: corteza de árboles y arbustos, troncos caídos, hojas, musgos y suelo. Xanthoria parietina es el taxón dominante en todas las zonas de recolección, con excepción del bosque relicto. Los géneros representados con el mayor número de especies son: Leptogium (8), Menegazzia (6), Pseudocyphellaria (19) y Ramalina (5).

En la Zona 1, la diversidad liquénica alcanza a 26 especies, gran parte de las cuales son comunes a las Zonas 2 y 3, con excepción de Pseudocyphellaria coriifolia, $P$. nitida y P. scabrosa. Los taxones Punctelia subrudecta y Pseudocyphellaria compar colonizan la mayoría de los troncos basales de las especies arbóreas del área.

En el bosque relicto (Zona 2) se registraron 47 taxones, que incluyen los líquenes foliícolas facultativos (Lücking et al. 2003) Psoroma caliginosum -en hojas de Laureliopsis philippiana-y Coenogonium luteum, en corteza de Aextoxicon punctatum. Pseudocyphellaria malmeana, especie rara conocida sólo en Chile y que ha sido recolectada en la Isla Mocha, en zonas aledañas a Valdivia y Chiloé (Galloway 1992) y en el Parque Nacional Queulat, Aisén, en el año 2008 (Quilhot et al. com.pers.), se registró en troncos basales de Aextoxicon punctatum; la frecuencia del taxón aumenta con la altura del hábitat, su área de mayor crecimiento estaría cercana al dosel del bosque. La mayoría de las especies de Sticta y de Leptogium se encuentran en esta zona de muestreo; las condiciones del hábitat al interior del bosque, en particular la escasa luminosidad y la elevada humedad ambiental, son requerimientos ecológicos de estas especies.

En el bosque dominado por Peumus boldus (Zona 3), se registró la mayor diversidad liquénica -52 taxones- que incluye a todas las especies de Menegazzia encontradas en la Isla Mocha. Las especies más comunes son Xanthoria parietina, Pseudocyphellaria berberina, P. compar, Punctelia subrudecta, Ramalina celastri, Rimelia reticulata, Teloschistes chrysophthalmus, $T$. flavicans y Usnea rubicunda.

Ramalina fastigiata -epífito de troncos y ramas en sitios abiertos- es un elemento subcosmopolita frecuente en zonas templadas de Europa, Asia y Norteamérica (Crespo \& Bueno 1984, Purvis et al. 1992, Wirth 1995). El taxón, que constituye un nuevo registro para Chile, se recolectó en la Zona 3 en troncos de Peumus boldus, donde forma comunidades con Ramalina celastri, R. chilena, R. chilensis y $R$. farinacea.

En la Zona 4, en rocas de la franja costera, sólo se registraron los taxones Candelaria concolor, Roccellina limitata y Xanthoria parietina.

La micobiota liquenizada de Isla Mocha es característica del bosque valdiviano; en este bioma se registraron especies de los géneros Collema, Flavoparmelia, Hypotrachyna, Leptogium, Menegazzia, Nephroma, Sticta, Pannaria, Pseudocyphellaria, Psoroma, Punctelia y Usnea.

Los taxones Coenogonium luteum, Dictyonema glabratum, Leptogium azureum, Menegazzia neozelandica, Nephroma antarcticum, N. plumbeum, Pseudocyphellaria berberina, P. coriifolia, P. crocata, P. dissimilis, P. flavicans, P. intricada, P.nitida, P. norvegica, P.santessonii, Roccellina limitata, Sticta lineariloba, Teloschistes flavicans, Usnea pusilla y Xanthoria parietina, que corresponden al $26 \%$ de las especies presentes en la Isla Mocha, se han registrado en el Archipiélago de Juan Fernández (Redon \& Quilhot 1977, 1983, Galloway 1992, 1994).

Los patrones biogeográficos resultan de procesos ecológicos que influencian la dispersión en variadas escalas de tiempo y de espacio (Wiens \& Donoghue 2004). La dispersión oceánica tiene un rol significativo en la generación de disyunciones y puede ocurrir con elevada frecuencia a mayores distancias que las esperadas (De Queiroz 2005), lo que explicaría la presencia de taxones de localidades muy distantes de la Isla Mocha, como ocurre con Pseudocyphellaria dissimilis y Sticta lineariloba considerados endémicos del archipiélago de Juan Fernández (Galloway 1992, 1994), y con las especies de Leptogium -L. brevissonii, L. britannicum y L. cochleatum- que sólo habían sido registradas en Chile en Laguna San Rafael, Aisén (Galloway \& Jorgensen 1995).

En la Isla Mocha predominan los elementos endémicos del sur de Sudamérica (38\%) -representados principalmente por especies de los géneros Pseudocyphellaria, Menegazzia y Sticta- y los elementos cosmopolitas (25\%) que incluyen especies de los géneros Candelaria, Flavoparmelia, Hypotrachyna, Peltigera, Physcia, Pseudocyphellaria, Punctelia, Teloschistes, Thelotrema, Usnea y Xanthoria. Los elementos tropicales, australes, australásicos, bipolares y otros, constituyen el $37 \%$ de la micobiota liquenizada de la Isla Mocha.

\section{CONCLUSIONES}

La flora liquénica en la Isla Mocha se distribuye en algunos sitios de la franja costera, en los bordes y el interior del bosque relicto. En la franja intermedia, las prácticas agrícolas y ganaderas han destruido el sustrato apropiado para el desarrollo de estos organismos; como por ejemplo de especies de los géneros Cladonia y Stereocaulon, característicos de sitios muy expuestos a la luz en el bosque valdiviano.

Desde una perspectiva liquenológica, el habitat más notable es el bosque relicto de A. punctatum, que data del Holoceno tardío (Le Quesne et al. 1999). Es probable que especies liquénicas relictas hayan persistido como, por ejemplo, Pseudocyphellaria malmeana que muestra 
una marcada preferencia por el sustrato -sólo se registró en troncos de $A$. punctatum- y/o por las características microclimáticas del hábitat tales como: escasa luminosidad, elevada humedad ambiental y protección frente a los vientos imperantes en la Isla.

Peumus boldus y Aextoxicon punctatum son las especies arbóreas más importantes para la conservación de la diversidad liquénica en este bioma.

\section{AGRADECIMIENTOS}

A la Dirección de Investigación Universidad de Valparaíso, DIPUV 27/2008. Al Sr. Mario Hahn y familia por su amistad y apoyo logístico.

\section{REFERENCIAS}

Adler, M. \& S. Calvelo. 1996. Two new species of the genus Menegazzia (Parmeliaceae sensu lato, lichenized Ascomycotina) from southern South America. Mycotaxon 59: 367-372.

Arvidsson, L. \& D.J. Galloway. 1992. Pannariaceae. Flora of Australia 54: 246-293.

BJERKE, J.W. 2001. A new sorediate species of Menegazzia (Parmeliaceae, lichenized Ascomycota) from Chile. Lichenologist 33: 117-120.

BJERKE, J.W. 2005. Synopsis of the lichen genus Menegazzia (Parmeliaceae.Ascomycota) in South America. Mycotaxon 91: 423-454.

BJerke, J.W., D.J. Galloway, A. Elvebakk \& W. Quilhot. 2003a. Pseudocyphellaria dasyphyllidia -a new phyllidiate species from Chile (Lobariaceae, lichenized Ascomycota). Cryptogamie, Mycologie 24: 59-66.

BJerke, J.W., A. Elvebakk \& W. Quilhot. 2003b. Distribution and habitat ecology of the sorediate species of Menegazzia (Parmeliaceae, lichenized Ascomycota) in Chile. Revista Chilena de Historia Natural 76: 79-98.

Crespo, A. \& A.G. Bueno. 1984. Flora liquénica epífita de Cádiz. I. Los alcornocales de las Sierras de Algeciras. Anales de Biología (Murcia) 1: 219-231.

De Queiroz, A. 2005. The resurrection of oceanic dispersal in historical biogeography. Trends in Ecology and Evolution 20: 68-73.

Dinerstein, E., D.M. Olson, D.J. Graham, A.L. Webster, S.A. Primm, M.P. Bookbinder \& G. Ledec. 1995. A conservation assessment of the terrestrial ecoregions of Latin America and the Caribean. The World Bank, Washington DC. 129 pp.

Elix, J.A. 1992. Hypogymnia. Flora of Australia 54: 201-213.

ElvebakK, A. \& J.W. BJerke. 2005. Pannaria isabellina (Vain.) Elvebakk \& Bjerke comb. nov., a remarkable lichen species from Chile. Lichenologist 37: 47-54.

ElvebakK, A. \& D.J. Galloway. 2003. Notes on the heterogenous genus Psoroma s. lat. in New Zealand. Australasian Lichenology 53: 4-9.

FiLson, R.B. 1992. Collema. Flora of Australia 54: 161-173.
Follmann, G. 1967. Die Flechtenflora der nordchilenischen Nebeloase Cerro Moreno. Nova Hedwigia 14: 215-281.

GallowaY, D.J. 1985. Flora of New Zealand Lichens. P.D. Hasselberg, Government Printer, Wellington, New Zealand. $662 \mathrm{pp}$.

Galloway, D.J. 1992. Studies in Pseudocyphellaria (lichens) III. The South American species. Bibliotheca Lichenologica 46: 1- 275.

Galloway, D.J. 1994. Studies on the lichen genus Sticta (Schreber) Ach.: I. Southern South American species. Lichenologist 26: 223-282.

Galloway, D.J. \& G. GuZmán. 1988. A new species of Phlyctis from Chile. Lichenologist 20: 393-397.

Galloway, D.J. \& P.M. Jørgensen. 1995. The lichen genus Leptogium (Collemataceae) in southern Chile. En: F.J.A. Daniels, M. Schultz \& J. Peine (eds.), Flechten Follmann. Contributions to Lichenology in Honour of Gerhard Follmann; pp. 227-247. Geobotanical and Phytotaxonomical Study Group. Botanical Institut, University of Cologne, Cologne.

Galloway, D.J. \& W. Quilhot. 1998. Checklist of Chilean lichenforming and lichenicolous fungi. Gayana Botanica 55: 111-185.

Galloway, D.J, R. Lewis Smith \& W. Quilhot. 2005. A new species of Placopsis (Agyriaceae: Ascomycota) from Antarctica. Lichenologist 37: 321-327.

Galloway, D.J., W. Quilhot \& P.M. Jørgensen. 2006. Pannaria conoplea and P. tavaresii (Ascomycota: Parmeliaceae) new to Chile. Lichenologist 38: 83-87.

Goward, T. \& T. AHTr.1992. Macrolichens and their zonal distribution in Wells Gray Provincial Park and its vicinity, British Columbia, Canada. Acta Botanica Fennica 147: 160.

Hale, M.E. 1975. A revision of the lichen genus Hypotrachyna (Parmeliaceae) in tropical America. Smithsionian Contributions to Botany 5: 1-73.

Hale, M.E. 1986. Flavoparmelia, a new genus in the lichen family Parmeliaceae (Ascomycotina). Mycotaxon 25: 603-605.

Hawksworth, D.L. 1988. A new name for Dictyonema pavonium (Swartz) Parmasto. Lichenologist 20: 101.

Hertel, H. 2007. Notes and records of Southern Hemisphere lecideoid lichens. En: I. Kärnefelt \& A. Thell (eds.), Lichenological Contributions in Honour of David Galloway. Bibliotheca Lichenologica 95: 267-296.

Jørgensen, P.M. 1998. Studies in the lichen family Pannariaceae VII. On some poorly known Parmeliella-like species from the Southern Hemisphere. Lichenologist 30: 533-541.

Jørgensen, P.M. 2004. Further contributions to the Pannariaceae (lichenized Ascomycetes) of the Southern Hemisphere. Bibliotheca Lichenologica 88: 229-253.

Jørgensen, P.M. \& M. Wedin. 1999. On Psoroma species from the Southern Hemisphere with cephalodia producing vegetative dispersal units. Lichenologist 31: 341-347.

Kalb, K., B. Staiget, J. Elix, U. Lange \& H.T. Lumbsh. 2008. Anew circumscription of the genus Ramboldia (Lecanoraceae, Ascomycota) based on morphological and molecular evidences. Nova Hedwigia 86: 23-42.

Kashiwadani, H. 1990. Some Chilean species of the genus Ramalina. Bulletin National Science Museum, Tokyo, Ser. B 16: 1-12. 
LAUNDON, J.R. 1981. The species of Chrysothrix. Lichenologist 13: 101-121.

Le Quesne, C., C. Villagrán \& R. Villa. 1999. Historia de los bosques relictos de "olivillo" (Aextoxicon punctatum) y Mirtáceas de la Isla Mocha, Chile, durante el Holoceno tardío. Revista Chilena de Historia Natural 72: 31-47.

LÜCKING, R. \& K. KaLB. 2000. Foliikole Flechten aus Brasilien (Vornehmlich Amazonien), inklusive einer Checkliste und Bemerkungen zu Coenogonium und Dimerella (Gyalectaceae). Botanische Jahrbücher für Systematik, Pflanzengeschichte und Pflanzengeographie 122:11-61.

Lücking, R., V. Wirth, L.I. Ferraro \& M.E.S. CÁceres. 2003. Foliicolous lichens from Valdivian temperate rain forests of Chile and Argentina: evidence of an austral element, with the description of seven new taxa. Global Ecology \& Biogeography 12: 21-36.

Martínez, I., A.R. Burgaz, O. Vitikainen \& A. Escudero. 2003. Distribution patterns in the genus Peltigera Willd. Lichenologist 35: 301-323.

Mendoza, G. \& V. Jerez. 2001. Coleópteros epígeos asociados a Peumus boldus Mol. en la Reserva Nacional Isla Mocha, Chile (Insecta, Coleoptera). Gayana 65: 129-136.

Messuti, M.I. 1996. Notes on the lichen genus Coccotrema in southern South America. New Zealand Journal of Botany 34: 57-64.

NAsh III, T.H. \& J.A. ElIx. 2002a. Flavoparmelia. In: T.H. Nash III, B.D. Ryan, C. Gries \& F. Bungartz (eds.), Lichen Flora of the Sonoran Desert Region, Vol. 1: 194-196. Lichens Unlimited, Arizona State University, Tempe, Arizona.

NASH III, T.H \& J.A. ElIx. 2002b. Rimelia. In: T.H. Nash III, B.D. Ryan, C. Gries \& F. Bungartz (eds.), Lichen Flora of the Sonoran Desert Region, Vol. 1: 449-451. Lichens Unlimited, Arizona State University, Tempe, Arizona.

Øvstedal, D.O. \& R.I. LewIS Smith. 2001. Lichens of Antarctica and South Georgia. A Guide to their Identification and Ecology. Cambridge University Press. 411 pp.

Parmasto, E. 1978. The genus Dictyonema (Thelephorolichenes). Nova Hedwigia 29: 99-144.

Pereira, I. 2007. Micobiota liquenizada del Parque Katalapi, X Región, Chile. Gayana Botanica 64: 192-200.

Pereira, I. \& W. Torres. 2005. Cinco nuevos registros de líquenes marinos para Chile. Gayana Botanica 62: 20-25.

Purvis, O.W., B.J. Coppins, D.L. Hawksworth, P.W. James \& D.M. Moore. 1992. Lichen flora of Great Britain and Ireland. London: Natural History Museum Publications. 710 pp.

Redon, J. \& W. Quilhot. 1977. Los líquenes de Juan Fernández. I. Estudio sistemático y ecológico preliminar. Anales Museo Historia Natural Valparaíso 10: 15-26.

Rivas Plata, E., R. LÜcking, A. Aptroot, H.J.M. Sipman, L. Chaves, J.L. Umaña \& D. Lizano. 2006. A first assessment of the Ticolichen biodiversity inventory in Costa Rica: the genus Coenogonium (Ostropales: Coenogoniaceae), with a world-wide key and checklist and a phenotype based cladistic analysis. Fungal Diversity 23: 255-321.

Tehler, A. 1983. The genera Dirina and Roccellina (Roccellaceae). Opera Botanica 70: 1-86.

Wetsberg, M. \& T.H. Nash III. 2002. Candelaria. In: T.H. Nash III, B.D. Ryan, C. Gries \& F. Bungartz (eds.), Lichen Flora of the Sonoran Desert Region. Vol. 1: 116-118. Lichens Unlimited, Arizona State University, Tempe, Arizona.

Wiens, J.J. \& M.J. Donoghue. 2004. Historical biogeography, ecology and species richness. Trends in Ecology and Evolution 19: 639-644.

White, F.J. \& P.W. JAMES. 1988. Studies on the genus Nephroma II. The southern temperate species. Lichenologist 20: 103166.

WIRTH, V. 1995. Die Flechten Baden-Württembergs. Teil 1. Ed. E. Ulmer. Stuttgart. 527 pp.

Recibido: 05.01.10

Aceptado: 05.07.10 